\title{
Transformation of Music Analysis and Teaching Ideas from the Perspective of Symbol Theory
}

\author{
Lin Cai \\ School of Music, Jiangxi Normal University, Nanchang, China \\ Email: 992514831@qq.com
}

How to cite this paper: Cai, L. (2019) Transformation of Music Analysis and Teaching Ideas from the Perspective of Symbol Theory. Open Access Library Journal, 6: e5655.

https://doi.org/10.4236/oalib.1105655

Received: July 31, 2019

Accepted: August 16, 2019

Published: August 19, 2019

Copyright $\odot 2019$ by author(s) and Open Access Library Inc.

This work is licensed under the Creative Commons Attribution International License (CC BY 4.0).

http://creativecommons.org/licenses/by/4.0/

(c) (7) Open Access

\begin{abstract}
Music, as a meaningful form, is a unique art created by human beings in order to realize the expression and communication of their inner life. Music analysis is a process of excavating, interpreting and understanding the potential meaning of creation. Combining the core concepts of symbolic theory with music text analysis, the symbolic narrative of music can be understood from the signifier and signified dimensions, thus presenting a new way and trend of meaning interpretation for music analysis and teaching concepts.
\end{abstract}

\section{Subject Areas}

Art, Education

Keywords

Symbol Theory, Music Analysis, Eero Tarasti, Intertexurality

\section{Introduction}

Since the 1980s, the development of New Musicology has been booming, gradually breaking the system definition of traditional music analysis disciplines, and entering the 1990s, especially the fusion of horizons and Intertextual Interpretation in the context of a new era culture with a more open and inclusive humanistic attitude. Using multi-disciplinary methods and perspectives, the analysis of works, which is the core of music research and teaching, is facing new opportunities and thinking changes. Obviously, compared with traditional music analysis, in the context of "new musicology", music analysis should be a broad, indefinite expression and independent choice with more cultural personality. It presents an unintegrated diversified and open understanding, but all the objectives are aimed at the meaning expression of music, which is shunned by traditional formalists and considered unspeakable. 


\section{Music Symbol Theory: Arising at the Historic Moment}

Although the dimension of music comprehension has been greatly broadened and extended, it should be noted that the more new integration in the multi-dimensional perspective, the more absolutely pure meta-linguistic meaning generation of music itself is required. The two seem to contradict each other, but in fact they are necessary for the exploration and long-cherished wish of the essence and unique expression of music. The transformation of research and teaching paradigm should not be conditioned by the opposite of "positivism" and the total negation. Although the fact-based scientific research method lacks the aesthetic and perceptual appeal of music art, rational exploration is an indispensable basic work. Only through music analysis can we obtain factual data and use them. Only by obtaining factual data through music analysis and compiling them for approval, can it be possible to explore the music history and music aesthetic humanistic situation which are close to "true" [1].

Music symbol theory seems to bridge the gap between form and content, representation structure and implicit ideas for a long time. It tries to find some rational rules to explain the perceptual meaning expression of music meta-language [2]. This may be questioned by some people, because they think that music aesthetic needs no real analysis and detailed explanation, only to appreciate the unique affect of music. However, for the development of music analysis research and teaching, seeking truth and meaning is an involuntary choice and mission. The uniqueness of the art of music lies in its clear two sides, one is the aesthetic expression of perception, the other is the logical composition of rationality, which is the natural combination of the two.

\section{Theoretical Interpretation of Musical Symbols}

Although the word "music symbol" is often visible, it is still quite obscure and strange to us, and music analysis and symbol theory have not yet realized the real fusion of ideas and cognitive generation.

As early as 1975, Jean-Jacques Nattiez introduced semiotics into music analysis as a methodology. He was devoted to the inductive bias and relationship establishment of musical symbol patterns. Although he introduced the semiotic approach, he focused the problem of musical symbols on the type of musical language itself, devoted himself to the summary of the analogical norms of rhetoric expression and musical language combination, and still confined his horizon to the musical language itself [3]. It can be seen that the theory of semiotics has not been integrated with music analysis, and has not been applied and updated with rich connotation and substance. They are still in a state of superficial correlation and internal separation.

Eero Tarasti changed this situation subversively. He accepted the classical semiotic tradition of Charles Sanders Peirce and Algirdas Julien Greimas. He had the idea of cultural structuralism and the thought of interdisciplinary citation. With a broad cultural perspective, he put non-verbal musical symbols back into 
the symbol domain and wrote measurable and analyzable narrative procedures and logical strategies such as time and space, existence and action [4]. He provides a philosophical analysis theory of music for the classic "triangle" problem of music history, form and meaning. Different from the inductive description of music behavior state, it follows the refined mathematical factor analysis and dynamic observation and development of space-time in music action, and understands the meaning of music which is hard to express by subtle changes of psychological modality and subject response [5], which undoubtedly has a subversive impact on traditional music analysis and teaching.

On the basis of his predecessors, Tarasti's analysis of musical symbols put forward a series of conceptions of musical meta-linguistic discourse patterns more systematically and clearly. More importantly, within the framework of this set of theoretical conceptions, Tarasti demonstrated the analysis process of musical works. His works were selected from the classical period to the 20th century to build up a musical understanding. A bridge between the real space of pitch structure and the meaning space of human metaphor. However, because of his rich German philosophical tradition and the influence of structuralism and existentialism in the 20th century, his theory of musical semiotics is quite profound. Therefore, before referring to and applying this set of music symbol analysis theory, we need to thoroughly absorb and understand the important concepts and methods.

\section{Music Analysis and Teaching Ideas from the Perspective of Symbol Theory}

Music is a meaningful form, a unique art form created by human beings in order to realize the expression and communication of their inner life. Therefore, the process of analyzing music works is the process of interpreting music, exploring its potential significance and then obtaining understanding.

\subsection{Reflections on Emphasizing Empirical Structure and Despising Critical Interpretation}

For a long time, music analysis teaching and application remain in a single skill level which is separated from aesthetic experience perception and historical and humanistic understanding. But this is not about ignoring technology. Artistic creation is man-made, not natural, and great art is like a natural, which is the result of the "game" between art and technology in the process of artists' creation. Therefore, analysis teaching should guide students to explain through analysis how artists use superb skills in the creative process to obtain freedom in the restriction. The most important thing is to use the means of technical analysis to know, taste, appreciate and understand music, so that more people are close to music and love music, rather than just understanding the technical means as a goal.

The traditional analysis of musical works is based on musical language ele- 
ments such as melody, tonality, rhythm, harmony and orchestration, which are dominated by the analysis of musical forms. It has long been formed as a model of graphic presentation. Despite illustrating the form and structure of music texts, there is a lack of interpretation of humanistic aesthetic significance, and it is difficult to appreciate the pulse of life and the spirit of the times expressed by music art under the cold analysis. Music acceptance and music analysis are in a split state. Innovation lies in the re-integration of the two. On the one hand, the symbolic discourse logic and the narrative procedure of the "action element" structure of the micro-music text, on the other hand, the influence of the macro-related social and cultural ideology on the generation of the meaning of the music text and the implicit implication. Therefore, the final analysis should be the interpretation of a meaningful form to gain the understanding of human nature and life.

\subsection{Context of "New Musicology" and Construction of "Discipline Core Quality"}

From the mastery of the basic knowledge of music analysis to the exploration of the spiritual soul of music analysis, that is, from "one-dimensional" basic skills to "three-dimensional" intellectual construction of content, form and purport, and then to "core quality" to cultivate "all-round development of people". It embodies the six qualities of humanities, scientific spirit, responsibility, practical innovation, learning to learn and healthy life. The change of educational concept reflects the change from subject-based to people-oriented, from external knowledge structure system to internal thinking of humanities consciousness. It attempts to explore and study the way of understanding the meaning of music in the context of the new era of cross-disciplines, and to present a unique way of expressing the meaning of music with effective analytical methods.

In music analysis and teaching, we need to understand and recognize the core concepts of symbol theory. The difficulty lies in mastering the theory and analysis procedure of musical symbols, and applying the symbolic narrative strategies of space-time and action dimensions such as "isotope", "combination-separation", "modality" and "symbolization generation process" to the analysis of musical works and the interpretation of humanistic significance, so as to provide operable understanding for the recognition of musical works.

\subsection{Historical Cognition and Experience Reading of Symbol Domain as a Cultural Sharer}

Of course, whether they are icons, indexes or symbols, music symbols will inevitably undergo re-evaluation and interpretation along with the shift of history and culture. The realization of the meaning expression of symbols requires that we have the ability to read, distinguish and explain various historical experiences and phenomena in the field of cultural semantics [6]. For example, in the interpretation of different textures in Baroque and Classical times, polyphonic music's linear form is an infinite yearning and the search for redemption. Its ap- 
pearance aims at enhancing the nobility of the counterpart text or pointing to the unknown divinity in the distance. On the contrary, classical music touches the earthly human consciousness, stresses the secular pleasure and places people at the center of everything. As a result, classical style liberates itself and enters a lively game world. As a special internal character, homophonic texture refers to the imaginary self. The melody is developed into various roles, provides human characteristics, and becomes a paradigm of musical discourse [7].

In traditional analysis, we often cannot feel the vivid vitality and moving charm of music, but only the stacking of technical terms and boring charts. In terms of musical form analysis, musical form is the time structure of music, while the objective physical time is different from the psychological time of music. If musical form analysis only divides the objective time stages, but does not see the vivid and expressive musical events that occur in the process of musical time, and does not interpret these events. Thus, such an analysis lacks intriguing experience of aesthetic significance.

\section{Conclusion}

It should be recognized that the necessary deconstruction and abstraction for analysis should not be forgotten or deviated from the original state of things. Analysis is a kind of recreation, that is to say, the analysts are first of all music receivers, whose feelings and understandings are naturally immersed in the aesthetic system of creation and communication. Simply technical analysis does not add new content to the aesthetic system, and the process of music symbolization and the construction of symbol domain will help music analysis get out of a single linguistic technology perspective and establish a multi-dimensional, intertextual meaning generation of correlation domain. It has positive enlightenment and reference for the diversification of music analysis teaching methods, the refinement and quantification of empirical analysis, and the exploration of the path of music meaning expression. It has practical guiding value for music performance and listening, music acceptance and communication, music teaching and appreciation, and other life and learning needs. For a long time, traditional cognition has turned to multi-interpretation of music aesthetics and humanistic experience in the context of the development of the new era, which is of great benefit and practical effect to the deep understanding of the humanistic quality of music art.

\section{Acknowledgements}

This work was supported by Jiangxi Social Science Planning General Project in 2018 (18YS05) and Jiangxi University Humanities Social Science Research Youth Project in 2018 (YS18104).

\section{Conflicts of Interest}

The author declares no conflicts of interest regarding the publication of this paper. 


\section{References}

[1] Hatten, R.S. (2004) Interpreting Musical Gesture, Topics, and Tropes: Mozart, Beethoven, Schubert. Indiana University Press, Bloomington.

[2] Cook, N. (1996) Putting the Meaning Back into Music, or Semiotics Revisited. $\mathrm{Mu}$ sic Theory Spectrum, 18, 106-123. https://doi.org/10.1525/mts.1996.18.1.02a00050

[3] Nattiez, J.-J. (1990) Music and Discourse: Toward a Semiology of Music. Princeton University Press, Princeton.

[4] Tarasti, E. (1994) A Theory of Musical Semiotics. Indiana University Press, Bloomington. https://doi.org/10.1515/9783110885187.585

[5] Tarasti, E. (2012) Semiotics of Classical Music: How Mozart, Brahms and Wagner Talk to Us. Deutsche Nationalbibliothek, Frankfurt?. https://doi.org/10.1515/9781614511410

[6] Kofi Agawu, V. (1991) Playing with Signs: A Semiotic Interpretation of Classic Music. Princeton University Press, Princeton. https://doi.org/10.1515/9781400861835

[7] Hatten, R. (1994) Musical Meaning in Beethoven: Markedness, Correlation, and Interpretation. Indiana University Press, Bloomington. 